\title{
Laplacian matching polynomial of graphs
}

\section{A. Mohammadian ${ }^{1}$}

Received: 12 November 2018 / Accepted: 27 May 2019 / Published online: 6 June 2019

C) Springer Science+Business Media, LLC, part of Springer Nature 2019

\begin{abstract}
In this article, we introduce the Laplacian matching polynomial of a graph. We prove some results concerning this new polynomial which can be viewed as analogue results on the classical matching polynomial.
\end{abstract}

Keywords Graph polynomial · Forest · Matching

Mathematics Subject Classification 05C31 05 C70

\section{Introduction}

Throughout this article, all graphs are assumed to be finite, undirected, and without loops or multiple edges. Let $G$ be a graph. The vertex set and the edge set of $G$ are denoted by $\mathcal{V}(G)$ and $\mathcal{E}(G)$, respectively. For a vertex $v$ of $G$, the degree of $v$, denoted by $\operatorname{deg}_{G}(v)$, is the number of edges of $G$ incident to $v$. The maximum degree of the vertices of $G$ is denoted by $\Delta(G)$. Let $S$ be a subset of $\mathcal{E}(G)$. We denote by $\mathcal{V}(S)$ the set of vertices of $G$ each of which is an endpoint of one of the edges in $S$. If no two distinct edges in $S$ share a common endpoint, then $S$ is called matching. The set of matchings of $G$ is denoted by $\mathcal{M}(G)$. A matching $M \in \mathcal{M}(G)$ with $\mathcal{V}(M)=\mathcal{V}(G)$ is named perfect matching. The matching polynomial of $G$ is defined as

$$
\mathscr{M}_{G}(x)=\sum_{M \in \mathcal{M}(G)}(-1)^{|M|} x^{|\mathcal{V}(G) \backslash \mathcal{V}(M)|} .
$$

The matching polynomial was introduced by Heilmann and Lieb in [3]. Many properties of the matching polynomial have been established. For instance, Heilmann and Lieb in [3] proved that all roots of $\mathscr{M}_{G}(x)$ are real and, if $\Delta(G) \geq 2$, then the roots of $\mathscr{M}_{G}(x)$ lie in the interval $(-2 \sqrt{\Delta(G)-1}, 2 \sqrt{\Delta(G)-1})$. Further, it is known that the

\footnotetext{
$凶$ A. Mohammadian ali_m@ahu.edu.cn

1 School of Mathematical Sciences, Anhui University, Hefei 230601, Anhui, China
} 
matching polynomial and the characteristic polynomial of $G$ are identical if and only if $G$ is a forest [7]. In this paper, we define the Laplacian matching polynomial of $G$ as

$$
\mathscr{L} \mathscr{M}_{G}(x)=\sum_{M \in \mathcal{M}(G)}(-1)^{|M|} \prod_{v \in \mathcal{V}(G) \backslash \mathcal{V}(M)}\left(x-\operatorname{deg}_{G}(v)\right) .
$$

We demonstrate that all roots of $\mathscr{L} \mathscr{M}_{G}(x)$ are real and, if $\Delta(G) \geq 2$, then the roots of $\mathscr{L} \mathscr{M}_{G}(x)$ lie in the interval $[0, \Delta(G)+2 \sqrt{\Delta(G)-1})$. Moreover, we show that the Laplacian matching polynomial and the Laplacian characteristic polynomial of $G$ are identical if and only if $G$ is a forest. The reader may discover some other properties of the Laplacian matching polynomial which can be viewed as analogue properties of the matching polynomial. For example, it is easily to check that if $G_{1} \cup G_{2}$ is the vertex disjoint union of two graphs $G_{1}$ and $G_{2}$, then $\mathscr{L} \mathscr{M}_{G_{1} \cup G_{2}}(x)=\mathscr{L} \mathscr{M}_{G_{1}}(x) \mathscr{L}_{\mathscr{M}} G_{2}(x)$.

Let us fix some notation and terminology. For a graph $G$, the adjacency matrix of $G$, denoted by $A(G)$, is a matrix whose rows and columns are indexed by $\mathcal{V}(G)$ and the $(u, v)$-entry is 1 if $u$ and $v$ are adjacent and 0 otherwise. Let $D(G)$ be the diagonal matrix whose rows and columns are indexed as the rows and the columns of $A(G)$ with $\operatorname{deg}_{G}(v)$ in the $v$ th diagonal position. The matrix $L(G)=D(G)-A(G)$ is said to be the Laplacian matrix of $G$. The Laplacian characteristic polynomial of $G$ is the characteristic polynomial of $L(G)$. Finally, for a subset $S$ of $\mathcal{V}(G)$, we denote the induced subgraph of $G$ on $S$ by $G[S]$.

\section{The roots}

In the following theorem, we prove that all roots of the Laplacian matching polynomial are nonnegative real numbers. Note that from Corollary 4 of [4] it follows that the Laplacian matching polynomial has no complex root. We consider an alternative approach to prove this fact whose idea comes from [6].

Theorem 2.1 For any graph $G$, all roots of $\mathscr{L}_{\mathscr{M}}(x)$ are real and nonnegative.

Proof Fix a graph $G$ on $n$ vertices and choose an arbitrary total order on $\mathcal{E}(G)$. A signing of the edges of $G$ is a $(-1,1)$-vector whose components are indexed by $\mathcal{E}(G)$. For a singing $s$, let $A_{s}(G)$ be the matrix whose rows and columns are indexed by $\mathcal{V}(G)$ and the $(u, v)$-entry is $\boldsymbol{s}_{u v}$ if $u$ and $v$ are adjacent and 0 otherwise. Let $L_{S}(G)=D(G)-A_{s}(G)$. Denote the set of permutations on a set $X$ by $\mathcal{S}_{X}$. By the expansion formula of the determinant and the linearity of the expectation, we have

$$
\begin{aligned}
\mathbb{E}_{S} & {\left[\operatorname{det}\left(x I-L_{S}(G)\right)\right] } \\
& =\mathbb{E}_{\boldsymbol{S}}\left[\sum_{\sigma \in \mathcal{S}_{\mathcal{V}(G)}} \operatorname{sign}(\sigma)\left(\prod_{v \in \mathcal{V}(G)}\left(x I-L_{S}(G)\right)_{v \sigma(v)}\right)\right] \\
& =\sum_{V \subseteq \mathcal{V}(G)}\left(\prod_{v \in \mathcal{V}(G) \backslash V}\left(x-\operatorname{deg}_{G}(v)\right)\right)\left(\sum_{\tau \in \mathcal{S}_{V}} \operatorname{sign}(\tau) \mathbb{E}_{\boldsymbol{S}}\left[\prod_{v \in V}\left(A_{\boldsymbol{S}}(G)\right)_{v \tau(v)}\right]\right) .
\end{aligned}
$$


In view of the definition of $A_{s}(G)$ and since the $\boldsymbol{s}_{u v}$ are independent random variables with $\mathbb{E}\left[\boldsymbol{s}_{u v}\right]=0$, only those products containing the squares of some of the $\boldsymbol{s}_{u v}$ have nonzero expectation value. Note that the expectation value of such products is 1 as $s_{u v}^{2}=1$. Moreover, we only consider those permutations on $V$ that are decomposed into mutually disjoint transpositions. These permutations represent the perfect matchings of $G[V]$. Therefore, we find from (1) that

$$
\begin{aligned}
\mathbb{E}_{s}\left[\operatorname{det}\left(x I-L_{s}(G)\right)\right] & =\sum_{V \subseteq \mathcal{V}(G)}\left(\prod_{v \in \mathcal{V}(G) \backslash V}\left(x-\operatorname{deg}_{G}(v)\right)\right)\left(\sum_{\substack{M \in \mathcal{M}(G[V]) \\
\mathcal{V}(M)=V}}(-1)^{|M|}\right) \\
& =\mathscr{L}_{\mathscr{M}_{G}}(x)
\end{aligned}
$$

For any $v \in \mathcal{V}(G)$, let $\boldsymbol{e}_{v}$ be the column vector whose components are indexed by $\mathcal{V}(G)$ with the $v$ th component being 1 and the other components being 0 . It is easy to check that

$$
L_{\boldsymbol{s}}(G)=\sum_{\{u, v\} \in \mathcal{E}(G)} \boldsymbol{\alpha}_{u v} \boldsymbol{\alpha}_{u v}^{\top}
$$

where

$$
\boldsymbol{\alpha}_{u v}= \begin{cases}\boldsymbol{e}_{u}-\boldsymbol{e}_{v} & \text { if } \boldsymbol{s}_{u v}=1 \\ \boldsymbol{e}_{u}+\boldsymbol{e}_{v} & \text { if } \boldsymbol{s}_{u v}=-1\end{cases}
$$

Notice that, in the definition of $\boldsymbol{\alpha}_{u v}$, we choose an arbitrary total order on $\mathcal{V}(G)$ and assume that $u$ precedes $v$. Using (2) and (3) and applying Theorem 4.1 of [5], we obtain that

$$
\begin{aligned}
\mathscr{L}^{\mathscr{M}} \mathscr{M}_{G}(x) & \mathbb{E}_{\boldsymbol{S}}\left[\operatorname{det}\left(x I-\sum_{\{u, v\} \in \mathcal{E}(G)} \boldsymbol{\alpha}_{u v} \boldsymbol{\alpha}_{u v}^{\top}\right)\right] \\
& =\left(\prod_{\{u, v\} \in \mathcal{E}(G)}\left(1-\frac{\partial}{\partial x_{u v}}\right)\right) \operatorname{det}\left(x I+\sum_{\{u, v\} \in \mathcal{E}(G)} x_{u v} \mathbb{E}\left[\boldsymbol{\alpha}_{u v} \boldsymbol{\alpha}_{u v}^{\top}\right]\right)_{\chi_{x_{u v}=0 \text { for all }\{u, v\} \in \mathcal{E}(G)}}
\end{aligned}
$$

Applying Proposition 2.4 of [1] and Corollary 3.8 and Proposition 3.9 of [5], we deduce that $\mathscr{L} \mathscr{M}_{G}(x)$ is a real stable polynomial. Hence, all roots of $\mathscr{L} \mathscr{M}_{G}(x)$ are real numbers. Furthermore, it follows from (3) that $L_{s}(G)$ is a positive semidefinite matrix and so $(-1)^{n} \operatorname{det}\left(x I-L_{s}(G)\right)>0$, for every signing $s$ and real number $x<0$. This along with (2) implies that $(-1)^{n} \mathscr{L} \mathscr{M}_{G}(x)=(-1)^{n} \mathbb{E}_{s}\left[\operatorname{det}\left(x I-L_{s}(G)\right)\right]>0$, for any $x<$ 0 . This means that all roots of $\mathscr{L} \mathscr{M}_{G}(x)$ are nonnegative, completing the proof. 
In what follows, we show that for any graph $G$ with $\Delta(G) \geq 2$, the roots of $\mathscr{L} \mathscr{M}_{G}(x)$ lie in the interval $[0, \Delta(G)+2 \sqrt{\Delta(G)-1})$. We first recall the following definition.

Definition 2.2 (Lass [4]) Let $G$ be a graph with $\mathcal{V}(G)=\left\{v_{1}, \ldots, v_{n}\right\}$. We define the multivariate matching polynomial of $G$ in the indeterminates $x_{1}, \ldots, x_{n}$ as

$$
\mathfrak{M}_{G}\left(x_{1}, \ldots, x_{n}\right)=\sum_{M \in \mathcal{M}(G)}(-1)^{|M|} \prod_{v_{i} \in \mathcal{V}(G) \backslash \mathcal{V}(M)} x_{i}
$$

The two following results on the multivariate matching polynomial can be established by similar proofs to that of the analogue results on the matching polynomial.

Proposition 2.3 Let $G$ be a graph with $\mathcal{V}(G)=\left\{v_{1}, \ldots, v_{n}\right\}$. For any $k \in\{1, \ldots, n\}$,

$$
\begin{aligned}
\mathfrak{M}_{G}\left(x_{1}, \ldots, x_{n}\right)= & x_{k} \mathfrak{M}_{G-v_{k}}\left(x_{1}, \ldots, \widehat{x_{k}}, \ldots, x_{n}\right) \\
& -\sum_{\left\{v_{k}, v_{\ell}\right\} \in \mathcal{E}(G)} \mathfrak{M}_{G-v_{k}-v_{\ell}}\left(x_{1}, \ldots, \widehat{x_{k}}, \ldots, \widehat{x_{\ell}}, \ldots, x_{n}\right) .
\end{aligned}
$$

Proof We have

$$
\begin{aligned}
& \mathfrak{M}_{G}\left(x_{1}, \ldots, x_{n}\right)=\sum_{\substack{M \in \mathcal{M}(G) \\
v_{k} \notin \mathcal{V}(M)}}(-1)^{|M|}\left(\prod_{v_{i} \in \mathcal{V}(G) \backslash \mathcal{V}(M)} x_{i}\right) \\
& +\sum_{\substack{M \in \mathcal{M}(G) \\
v_{k} \in \mathcal{V}(M)}}(-1)^{|M|}\left(\prod_{v_{i} \in \mathcal{V}(G) \backslash \mathcal{V}(M)} x_{i}\right) \\
& =x_{k} \sum_{M \in \mathcal{M}\left(G-v_{k}\right)}(-1)^{|M|}\left(\prod_{v_{i} \in \mathcal{V}\left(G-v_{k}\right) \backslash \mathcal{V}(M)} x_{i}\right) \\
& +\sum_{\left\{v_{k}, v_{\ell}\right\} \in \mathcal{E}(G)} \sum_{\substack{M \in \mathcal{M}(G) \\
\left\{v_{k}, v_{\ell}\right\} \in M}}(-1)^{|M|}\left(\prod_{v_{i} \in \mathcal{V}(G) \backslash \mathcal{V}(M)} x_{i}\right) \\
& =x_{k} \mathfrak{M}_{G-v_{k}}\left(x_{1}, \ldots, \widehat{x_{k}}, \ldots, x_{n}\right) \\
& -\sum_{\left\{v_{k}, v_{\ell}\right\} \in \mathcal{E}(G)} \sum_{M \in \mathcal{M}\left(G-v_{k}-v_{\ell}\right)}(-1)^{|M|}\left(\prod_{v_{i} \in \mathcal{V}\left(G-v_{k}-v_{\ell}\right) \backslash \mathcal{V}(M)} x_{i}\right) \\
& =x_{k} \mathfrak{M}_{G-v_{k}}\left(x_{1}, \ldots, \widehat{x_{k}}, \ldots, x_{n}\right) \\
& -\sum_{\left\{v_{k}, v_{\ell}\right\} \in \mathcal{E}(G)} \mathfrak{M}_{G-v_{k}-v_{\ell}}\left(x_{1}, \ldots, \widehat{x_{k}}, \ldots, \widehat{x_{\ell}}, \ldots, x_{n}\right) \text {. }
\end{aligned}
$$


Theorem 2.4 Let $G$ be a graph with $\Delta(G) \geq 2$. Then $\mathfrak{M}_{G}\left(x_{1}, \ldots, x_{n}\right)>0$ for every real numbers $x_{1}, \ldots, x_{n} \geq 2 \sqrt{\Delta(G)-1}$.

Proof For every given graph $G$ with $\mathcal{V}(G)=\left\{v_{1}, \ldots, v_{n}\right\}$, integer $k \geq \max \{\Delta(G), 2\}$, and real numbers $x_{1}, \ldots, x_{n} \geq 2 \sqrt{k-1}$, we prove by induction on $n$ that

$$
\mathfrak{M}_{G}\left(x_{1}, \ldots, x_{n}\right)>\sqrt{k-1} \mathfrak{M}_{G-v_{i}}\left(x_{1}, \ldots, \widehat{x_{i}}, \ldots, x_{n}\right)
$$

provided $\operatorname{deg}_{G}\left(v_{i}\right)<k$. Using Proposition 2.3 and the induction hypothesis, we have

$$
\begin{aligned}
\frac{\mathfrak{M}_{G}\left(x_{1}, \ldots, x_{n}\right)}{\mathfrak{M}_{G-v_{i}}\left(x_{1}, \ldots, \widehat{x_{i}}, \ldots, x_{n}\right)} & =x_{i}-\sum_{\left\{v_{i}, v_{j}\right\} \in \mathcal{E}(G)} \frac{\mathfrak{M}_{G-v_{i}-v_{j}}\left(x_{1}, \ldots, \widehat{x_{i}}, \ldots, \widehat{x_{j}}, \ldots, x_{n}\right)}{\mathfrak{M}_{G-v_{i}}\left(x_{1}, \ldots, \widehat{x_{i}}, \ldots, x_{n}\right)} \\
& >2 \sqrt{k-1}-\frac{k-1}{\sqrt{k-1}}=\sqrt{k-1}
\end{aligned}
$$

as we wanted.

Now, for any graph $G$ with $\mathcal{V}(G)=\left\{v_{1}, \ldots, v_{n}\right\}$ and $\Delta(G) \geq 2$, we prove by induction on $n$ that $\mathfrak{M}_{G}\left(x_{1}, \ldots, x_{n}\right)>0$ for every real numbers $x_{1}, \ldots, x_{n} \geq$ $2 \sqrt{\Delta(G)-1}$. By Proposition 2.3 and applying what we proved in the previous paragraph for $\Delta(G)$ instead of $k$, we find for some $i$ that

$$
\begin{aligned}
\frac{\mathfrak{M}_{G}\left(x_{1}, \ldots, x_{n}\right)}{\mathfrak{M}_{G-v_{i}}\left(x_{1}, \ldots, \widehat{x_{i}}, \ldots, x_{n}\right)} & =x_{i}-\sum_{\left\{v_{i}, v_{j}\right\} \in \mathcal{E}(G)} \frac{\mathfrak{M}_{G-v_{i}-v_{j}}\left(x_{1}, \ldots, \widehat{x_{i}}, \ldots, \widehat{x_{j}}, \ldots, x_{n}\right)}{\mathfrak{M}_{G-v_{i}}\left(x_{1}, \ldots, \widehat{x_{i}}, \ldots, x_{n}\right)} \\
& >2 \sqrt{\Delta(G)-1}-\frac{\Delta(G)}{\sqrt{\Delta(G)-1}}=\frac{\Delta(G)-2}{\sqrt{\Delta(G)-1}} .
\end{aligned}
$$

It follows from $\Delta(G) \geq 2$ and the induction hypothesis that $\mathfrak{M}_{G}\left(x_{1}, \ldots, x_{n}\right)>0$.

Corollary 2.5 Let $G$ be a graph with $\Delta(G) \geq 2$. Then $\mathscr{L} \mathscr{M}_{G}(x) \neq 0$ for any real number $x \geq \Delta(G)+2 \sqrt{\Delta(G)-1}$.

Proof Let $\mathcal{V}(G)=\left\{v_{1}, \ldots, v_{n}\right\}$ and consider a real number $x \geq \Delta(G)+$ $2 \sqrt{\Delta(G)-1}$. Since the numbers $x-\operatorname{deg}_{G}\left(v_{1}\right), \ldots, x-\operatorname{deg}_{G}\left(v_{n}\right)$ are at least $2 \sqrt{\Delta(G)-1}$, it follows from Theorem 2.4 that $\mathscr{L}_{\mathscr{M}}(x)=\mathfrak{M}_{G}\left(x-\operatorname{deg}_{G}\left(v_{1}\right), \ldots\right.$, $\left.x-\operatorname{deg}_{G}\left(v_{n}\right)\right)>0$.

The statement of the next theorem is obtained by combining Theorem 2.1 and Corollary 2.5 .

Theorem 2.6 For any graph $G$, all roots of $\mathscr{L}_{\mathscr{M}_{G}}(x)$ are nonnegative real numbers. Moreover, the roots of $\mathscr{L} \mathscr{M}_{G}(x)$ are contained in the interval $[0, \Delta(G)+$ $2 \sqrt{\Delta(G)-1})$ provided $\Delta(G) \geq 2$. 


\section{Forests}

In this section, we demonstrate that the Laplacian matching polynomial and the Laplacian characteristic polynomial of a graph $G$ are identical if and only if $G$ is a forest. We first recall the two following well-known theorems.

Theorem 3.1 (Harary [2]) Let $G$ be a graph on $n$ vertices. Then

$$
\operatorname{det}(A(G))=(-1)^{n} \sum_{H}(-1)^{p(H)} 2^{q(H)},
$$

where the summation runs over all spanning subgraphs of $G$ whose connected components are either $K_{2}$ or a cycle, $p(H)$ denotes the number of connected components of $H$, and $q(H)$ denotes the number of cycles of $H$.

Theorem 3.2 The perfect matching in each forest is unique if there exists one.

Theorem 3.3 Let G be a graph. The Laplacian matching polynomial and the Laplacian characteristic polynomial of $G$ are identical if and only if $G$ is a forest.

Proof By the expansion formula of the determinant, we may write

$$
\operatorname{det}(x I-L(G))=\sum_{S \subseteq \mathcal{V}(G)} \operatorname{det}(A(G[S]))\left(\prod_{v \in \mathcal{V}(G) \backslash S}\left(x-\operatorname{deg}_{G}(v)\right)\right)
$$

First, assume that $G$ is a forest. By Theorem 3.1,

$$
\operatorname{det}(A(G[S]))= \begin{cases}(-1)^{\frac{|S|}{2}} & \text { if } G[S] \text { has a perfect matching } \\ 0 & \text { otherwise. }\end{cases}
$$

Using (1) and Theorem 3.2, it is easily seen that $\operatorname{det}(x I-L(G))=\mathscr{L} \mathscr{M}_{G}(x)$, as desired. Now, assume that $G$ has a cycle. Let $g$ be the girth of $G$. By Theorems 3.1 and 3.2, we have

$$
\begin{aligned}
& \sum_{\substack{S \subseteq \mathcal{V}(G) \\
|S|<g}} \operatorname{det}(A(G[S]))\left(\prod_{v \in \mathcal{V}(G) \backslash S}\left(x-\operatorname{deg}_{G}(v)\right)\right) \\
& =\sum_{\substack{M \in \mathcal{M}(G) \\
|\mathcal{V}(M)|<g}}(-1)^{|M|}\left(\prod_{v \in \mathcal{V}(G) \backslash \mathcal{V}(M)}\left(x-\operatorname{deg}_{G}(v)\right)\right) .
\end{aligned}
$$

If we denote the polynomial given in (5) by $\varphi(x)$, then it follows from (1) and (4) that the coefficients of $x^{n-g}$ in $\mathscr{L}_{\mathscr{M}}{ }_{G}(x)-\varphi(x)$ and $\operatorname{det}(x I-L(G))-\varphi(x)$ are

$$
\sum_{\substack{M \in \mathcal{M}(G) \\|\mathcal{V}(M)|=g}}(-1)^{|M|} \text { and } \sum_{\substack{S \subseteq \mathcal{V}(G) \\|S|=g}} \operatorname{det}(A(G[S]))
$$


respectively. Again, by Theorems 3.1 and 3.2, we deduce that

$$
\left|\sum_{\substack{M \in \mathcal{M}(G) \\|\mathcal{V}(M)|=g}}(-1)^{|M|}-\sum_{\substack{S \subseteq \mathcal{V}(G) \\|S|=g}} \operatorname{det}(A(G[S]))\right|
$$

is twice of the number of cycles of the length $g$ in $G$. This means that $\mathscr{L} \mathscr{M}_{G}(x) \neq$ $\operatorname{det}(x I-L(G))$.

The following result is a consequence of Corollary 2.5 and Theorem 3.3. The original proof is complicated and uses a different method.

Theorem 3.4 (Stevanović [8]) For each forest $F$ with $\Delta(F) \geq 2$, the eigenvalues of $L(F)$ are less than $\Delta(F)+2 \sqrt{\Delta(F)-1}$.

Acknowledgements The author would like to thank two anonymous referees for their helpful comments.

\section{References}

1. Borcea, J., Brändén, P.: Applications of stable polynomials to mixed determinants: Johnson's conjectures, unimodality, and symmetrized Fischer products. Duke Math. J. 143, 205-223 (2008)

2. Harary, F.: The determinant of the adjacency matrix of a graph. SIAM Rev. 4, 202-210 (1962)

3. Heilmann, O.J., Lieb, E.H.: Theory of monomer-dimer systems. Comm. Math. Phys. 25, 190-232 (1972)

4. Lass, B.: Mehler formulae for matching polynomials of graphs and independence polynomials of clawfree graphs. J. Combin. Theory Ser. B 102, 411-423 (2012)

5. Marcus, A.W., Spielman, D.A., Srivastava, N.: Interlacing families II: mixed characteristic polynomials and the Kadison-Singer problem. Ann. of Math. (2) 182, 327-350 (2015)

6. Marcus, A.W., Spielman, D.A., Srivastava, N.: Interlacing families I: bipartite Ramanujan graphs of all degrees. Ann. of Math. (2) 182, 307-325 (2015)

7. Sachs, H.: Beziehungen zwischen den in einem Graphen enthaltenen Kreisen und seinem charakteristischen Polynom. Publ. Math. Debrecen 11, 119-134 (1964)

8. Stevanović, D.: Bounding the largest eigenvalue of trees in terms of the largest vertex degree. Linear Algebra Appl. 360, 35-42 (2003)

Publisher's Note Springer Nature remains neutral with regard to jurisdictional claims in published maps and institutional affiliations. 